REGARDS

SUR L'ECONOMIE ALLEMANDE

BULLETIN ECONOMIQUE DU CRAC
Regards sur l'économie allemande

Bulletin économique du CIRAC

115 | 2014

Varia

\title{
Vers une réforme de fond du fédéralisme financier
}

Isabelle Bourgeois

\section{OpenEdition}

Journals

Édition électronique

URL : http://journals.openedition.org/rea/4763

DOI : 10.4000/rea.4763

ISSN : 1965-0787

Éditeur

CIRAC

Édition imprimée

Date de publication : 1 décembre 2014

Pagination : 17-30

ISSN : 1156-8992

Référence électronique

Isabelle Bourgeois, «Vers une réforme de fond du fédéralisme financier 》, Regards sur l'économie

allemande [En ligne], 115 | décembre 2014, mis en ligne le 01 décembre 2016, consulté le 05 mai 2019. URL : http://journals.openedition.org/rea/4763 ; DOI : 10.4000/rea.4763

(C) CIRAC 


\section{Vers une réforme de fond du fédéralisme financier}

\section{Isabelle Bourgeois}

La réforme du fédéralisme financier engagée à la fin de l'été comptera, si elle ne se limite pas à quelques aménagements comme par le passé, parmi les réformes majeures menées en République fédérale et les réformes modèles menées dans le fil de l'intégration européenne. Plusieurs éléments se conjuguent pour expliquer que cette réforme, dont l'intention figure dans le Contrat de coalition signé en 2012 par CDU/CSU et SPD, soit effectivement mise en chantier.

Des éléments de calendrier, d'abord : le Pacte de solidarité II (Solidarpakt II) au titre duquel sont versées les aides à la reconstruction des Länder est-allemands expire le 31 décembre 2019 ; la loi régissant les modalités de la péréquation financière horizontale entre les Länder expire à la même date. Des éléments de politique budgétaire interne, ensuite : la "règle d'or » inscrite dans la Loi fondamentale exige des Länder que leurs budgets soient à l'équilibre dès l'exercice 2020. Or certains d'entre eux, tout particulièrement Berlin, sont lourdement endettés et ne parviendront pas à respecter ce délai sans aide. Un motif de politique européenne, enfin : le Traité TSCG, c'est-à-dire le pacte budgétaire par lequel les Etats de la zone Euro s'engagent à réduire leur endettement, est entré

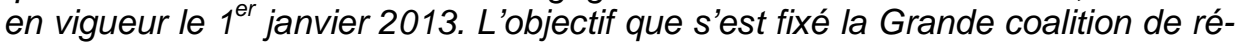
duire le taux de la dette allemande à moins de $70 \%$ du PIB d'ici la fin 2017 ne pourra être atteint que par un effort collectif de modernisation en profondeur de la répartition des ressources comme des compétences au sein d'un fédéralisme rénové. C'est ainsi que l'Allemagne compte à la fois pérenniser les fondements de sa compétitivité et assumer la responsabilité qui lui incombe dans l'UE : apporter, "grâce à une politique budgétaire axée sur la stabilité et la croissance et menée à tous les échelons de l'Etat, sa contribution à la stabilité de la zone Euro » (Contrat de coalition). Des travaux herculéens...

\section{Une réforme en profondeur s'impose depuis longtemps}

II ne s'agit nullement, pour l'Allemagne, de remettre en question l'organisation fédérale de ses finances publiques. Le fédéralisme financier fait partie intégrante du fédéralisme politique en ce sens qu'il confie à chacun des acteurs les moyens de donner corps à l'équilibre entre les principes de subsidiarité (autonomie) et de solidarité collective sur lesquels repose l'organisation de l'Etat, c'est-à-dire en l'occurrence de cette " communauté solidaire » (Tribunal constitutionnel fédéral, 2 BvF, 2/98) qu'est la République fédérale d'Allemagne. Les règles de sa "Constitution financière " (Finanzverfassung) visent donc à la fois à respecter les identités des Etats membres de la RFA que sont les Länder et à assurer un certain degré de convergence des politiques économiques et budgétaires au sein de l'Allemagne. Ces bases-là sont immuables.

II s'agit 'simplement' aujourd'hui de rendre plus efficient le fonctionnement de ce modèle d'organisation en articulant mieux les compétences et missions des différents échelons avec les moyens respectivement disponibles. II convient pour cela de " trouver le juste milieu entre d'un côté l'indépendance, la responsabilité et la préservation de l'individualité des Länder et, de l'autre, leur co-responsabilité, dans un collectif solidaire, pour l'existence et l'autonomie de leurs partenaires dans la fédération ". Voilà l'un des principes directeurs de réforme qu'avait formulés le Tribunal constitutionnel fédéral dans un grand arrêt rendu

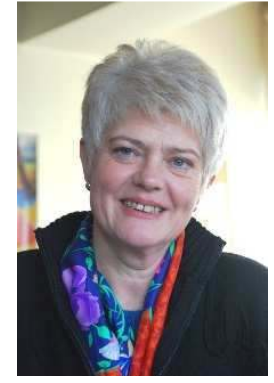

Isabelle Bourgeois, rédactrice en chef de Regards sur l'économie allemande

Modernisation interne et adaptation aux règles de la zone Euro 
Länder : pouvoir administratif et autonomie budgétaire

Trop de transferts ont abouti au nivellement par le bas

Des impôts revenant de droit aux trois échelons territoriaux...

... et des impôts communs partagés entre eux en novembre 1999 à propos du Länderfinanzausgleich : la péréquation financière horizontale (inter-étatique) entre les Länder qui est l'un des principaux instruments de répartition de cet objectif de convergence (2 BvF 2/98, al. 291). II s'agit encore plus aujourd'hui de moderniser aussi ce système pour mieux l'adapter aux règles de fonctionnement de la zone Euro.

La Fédération (Bund) et les 16 Länder qui la constituent resteront donc autonomes et indépendants au plan budgétaire ; actuellement, le Bund maîtrise moins de la moitié du budget global allemand. II y aura donc toujours $16+1$ budgets publics indépendants en Allemagne, correspondant aux tâches et missions revenant à chacun de ces échelons dans le partage des compétences prévu par le fédéralisme politique (voir REA 102/ 2011). Si les Länder ont peu de pouvoirs propres dans le partage des compétences sectorielles, législatives (leur souveraineté se limite à la culture et à l'enseignement), il n'en va pas de même dans le partage fonctionnel des pouvoirs : ils sont en effet seuls souverains en matière de pouvoir administratif ; celui du Bund se limite aux affaires étrangères, à l'armée et aux douanes, et il n'y a donc pas en Allemagne, à l'exception de ces trois domaines, d'administration centrale telle que nous la connaissons en France. Ce sont les Länder qui donnent corps sur leur territoire aux lois adoptées à l'échelon fédéral, et ce sont eux par exemple qui gèrent et rémunèrent les personnels publics qu'ils emploient.

Le même pouvoir administratif les habilite à mener chacun sa propre politique économique: la responsabilité budgétaire en est le corollaire constitutionnel. Mais ils exercent leur pouvoir et missions à l'intérieur d'un cadre de politique générale dont les grandes lignes sont fixées conjointement à l'échelon fédéral grâce à l'articulation des pouvoirs législatifs entre Bundestag et Bundesrat. Leur autonomie administrative et budgétaire leur donne donc les moyens de mener leur propre politique structurelle (régionale). Cette diversité - et concurrence des approches politiques et la polyarchie qui en est une condition sont l'un des facteurs systémiques de la compétitivité allemande (Bourgeois/Lasserre, 2011). Du moins en théorie, car depuis longtemps, dans la pratique, la solidarité qui est l'autre principe sur lequel repose le modèle allemand du fédéralisme visant à corriger les excès potentiels de la concurrence, et qui se traduit au plan financier par un flux important de transferts, a connu au fil des décennies une dérive égalitaire. Celle-ci a abouti à un «nivellement » par le bas, constaté en 1999 par le Tribunal constitutionnel fédéral, qui bride au contraire le potentiel de compétitivité des Länder et de l'Allemagne dans son ensemble, tout comme il grève les finances publiques de tous.

En effet, l'autonomie budgétaire des Länder se limite aux dépenses liées à l'exécution de leurs missions. Chaque Land finance lui-même sa politique mais il n'a guère la maîtrise de ses recettes. Certes, certains types d'impôts sont classés selon l'échelon territorial qui les prélève et auquel ils reviennent de droit. Ainsi, la taxe sur les activités industrielles et commerciales ou l'impôt foncier reviennent de droit aux communes, dont les finances sont largement autonomes de celles de leur Land ; l'impôt sur les successions ou le patrimoine, aux Länder ; les droits d'accises sur l'alcool (sauf la bière), le tabac et l'énergie, ou les taxes sur les assurances, au Bund. Ainsi réparties par lieu (entité territoriale) de perception, les recettes propres des communes s'élèvent à 84,5 milliards $€$, celles des Länder à 15,7 milliards $€$ et celles du Bund à un peu plus de 100 milliards $€$ en 2013 (ministère fédéral des Finances).

Le fédéralisme allemand étant de type coopératif, parallèlement à ce régime d'attribution de ces ressources fiscales spécifiques selon les échelons de souveraineté (Trennsystem), le système fiscal prévoit une deuxième catégorie : celle des impôts communs (Gemeinschaftssteuern) dont l'encours est partagé entre Bund, Länder et communes. II s'agit de l'impôt sur le revenu, de la taxe sur les transactions financières et les plus-values, de l'impôt sur les sociétés et de la TVA - autrement dit : de quelque $70 \%$ des recettes fiscales dont le total, toutes catégories confondues, atteignait près de 620 milliards $€$ en 2013. Le ba- 
rème de ces prélèvements est uniforme dans toute l'Allemagne, quel que soit le lieu de perception. Au total, les recettes de ces seuls impôts communs s'élevaient à un peu plus de 442,7 milliards $€$ en 2013. Ces chiffres donnent une idée de l'ampleur du chantier de réforme ...

Fédéralisme financier allemand : un système de répartition à 4 étages

L'encours des impôts communs (619,7 milliards $€$ en 2013 ; ministère fédéral des Finances) est réparti entre Bund, Länder et communes selon plusieurs critères et en 4 étapes ou étages successifs.

$1^{\text {er }}$ étage : répartition verticale entre Bund, Länder et communes selon la clé suivante (2013) :

Impôt sur le revenu

Impôt sur les sociétés

Taxe sur les transactions financières et les plus-values

TVA

$\begin{array}{llc}\text { Bund } & \text { Länder } & \text { Communes } \\ 42,5 \% & 42,5 \% & 15 \% \\ 50 \% & 50 \% & - \\ 44 \% & 44 \% & 12 \% \\ 53,5 \% & 44,5 \% & 2 \%\end{array}$

$2^{\mathrm{e}}$ étage : répartition horizontale entre les Länder.

Les Länder distribuent entre eux le volume global des impôts leur revenant collectivement.

IR : à chaque entité - Land, commune - revient de droit le produit des impôts perçus sur son territoire. Mais ce principe du lieu de perception est corrigé d'une ventilation visant à refléter le nombre de contribuables de chaque entité, qu'ils vivent sur le lieu de perception ou non.

IS : principe du lieu de perception, mais ventilé en fonction des entités territoriales où sont implantés les sites de production de la société concernée.

Taxe sur les transactions financières et les plus-values : principe du lieu de perception.

TVA : deux étapes et critères de répartition du volume global (les 44,5\%, soit 99,3 milliards € en 2013) :

- $25 \%$ sont retenus pour alimenter une quote-part compensatoire attribuée selon une grille linéaire progressive à ceux des Länder dont le montant par tête des recettes issues des impôts propres du Land et de sa part des impôts communs (hors TVA) est inférieur à la moyenne des Länder ;

- $75 \%$ de ce volume sont répartis en fonction du nombre d'habitants de chaque Land.

\section{$3^{\mathrm{e}}$ étage : péréquation horizontale entre les Länder (Länderfinanzausgleich).}

Cette péréquation, expression de la solidarité inter-étatique qui lie les Länder entre eux, lisse les disparités entre leurs capacités financières calculées par tête : somme des recettes du Land $+64 \%$ des recettes des communes sur leur territoire. En 2013, le total des montants transférés s'élevait à près de 8,5 milliards $€$.

Les trois Länder contributeurs sont la Bavière (4 320 mrds €) le Bade-Wurtemberg (2 429) et la Hesse (1 711).

Les 13 Länder bénéficiaires sont : Berlin (3 328), la Saxe (1 002), la Rhénanie du Nord-Westphalie (693), Brême (589), la Saxe-Anhalt (563), la Thuringe (547), le Brandebourg (521), le Mecklembourg (464), la Rhénanie-Palatinat (243), le Schleswig-Holstein (169), la Sarre (138), la Basse-Saxe (106) et Hambourg (87).

$4^{\mathrm{e}}$ étage (vertical) : dotations compensatoires du Bund à certains Länder.

Le Bund lisse les disparités restantes en versant aux Länder à capacité financière inférieure des dotations compensatoires (Bundesergänzungszuweisungen) d'un montant de près de 11 milliards $€$ en 2013 . Deux cas sont prévus : - cas général : ce dispositif vise à hisser la capacité financière de chaque Land à 99,5\% de la variable d'ajustement, c'est-à-dire d'une moyenne de tous les Länder (3,2 milliards $€$ ) ;

- cas particulier : aides exceptionnelles (non affectées) destinées à soutenir les budgets des Länder confrontés à des charges ou besoins particuliers : frais de gouvernance politique jugés supérieurs à la moyenne (10 Länder concernés) ; dotation versée aux nouveaux Länder pour couvrir les charges exceptionnelles liées à leur fort taux de chômage dans le cadre de la transition ; aide aux nouveaux Länder (dans le cadre du Pacte de solidarité II) notamment pour mettre à niveau leurs infrastructures. Total en $2013: 7,8$ milliards $€$.

Capacité financière des Länder (en \% de la moyenne) avant et après les principaux mécanismes de redistribution

\begin{tabular}{|c|c|c|c|c|c|c|c|c|c|c|c|c|c|c|c|}
\hline B-W & B-S & BAV & BE & BB & HB & HH & HE & M-P & R-P & NRW & SAR & SAX & S-AN & S-H & TH \\
\hline \multicolumn{16}{|c|}{ Etage 2} \\
\hline \multicolumn{16}{|c|}{ Après répartition verticale Bund/Länder de la part de l'IR et de l'IS revenant aux Länder } \\
\hline \multicolumn{16}{|c|}{ Après régime général de répartition de la TVA (75 \% du total revenant aux Länder) } \\
\hline 86,9 & 103,8 & 86,9 & 98,1 & 135,9 & 103,6 & 86,9 & 86,9 & 152,9 & 92,5 & 87,6 & 116,0 & 153,3 & 152,9 & 95,1 & 154,0 \\
\hline \multicolumn{16}{|c|}{ Après attribution de la quote-part de TVA (ajustement des $25 \%$ restants)) } \\
\hline \multicolumn{16}{|c|}{ Etage 3} \\
\hline \multicolumn{16}{|c|}{ Après péréquation horizontale entre les Länder (Länderfinanzausgleich) : 8,5 milliards $€$} \\
\hline 104,4 & 99,5 & 105,7 & 90,7 & 96,0 & 91,3 & 98,8 & 105,0 & 95,2 & 98,2 & 98,8 & 96,9 & 95,6 & 95,6 & 98,3 & 95,6 \\
\hline \multicolumn{16}{|c|}{ Etage 4} \\
\hline
\end{tabular}

Source des données : Bundesministerium der Finanzen, Monatsbericht, février 2014. Etat : 2013. B-S : Basse-Saxe ; B-W : Bade-Wurtemberg ; BAV : Bavière; BE : Berlin; BB : Brandebourg; HB : Brême; HH : Hambourg; HE : Hesse ; M-P : Mecklembourg-Poméranie occidentale ; R-P : RhénaniePalatinat ; NRW : Rhénanie du Nord-Westphalie ; SAR : Sarre ; SAX : Saxe ; S-AN : Saxe-Anhalt ; S-H : Schleswig-Holstein ; TH : Thuringe. 
L'absence de maîtrise des recettes incite les Länder à s'endetter

Accroître leu responsabilité budgétaire

La solidarité généralisée et indifférenciée, est contre-productive

Trop de dépenses mixtes
Le budget de chaque Land est donc abondé majoritairement grâce au partage horizontal et vertical à la fois - entre le Bund et les Länder du produit de ces impôts communs. Autrement dit, pour financer l'essentiel de sa politique économique (et notamment le développement des infrastructures régionales), un Land ne dispose que de deux principales sources: d'un côté ses recettes propres, augmentées de la part des recettes fiscales communes qui lui revient, et à quoi s'ajoutent le plus souvent des dotations compensatoires du Bund, et de l'autre côté, le recours à l'endettement. C'est cette définition quelque peu réductrice de l'autonomie budgétaire qui est à l'origine par exemple de la lourde dette contractée par les Länder de Berlin, Brème, de Sarre ou de Hambourg.

Dette des Länder par habitant (2012)

\begin{tabular}{|lr|lr|}
\hline Bade-Wurtemberg & $4032 €$ & Mecklembourg-Poméranie occid. & $5926 €$ \\
Basse-Saxe & $7108 €$ & Rhénanie-Palatinat & $8062 €$ \\
Bavière & $2250 €$ & Rhénanie du Nord-Westphalie & $7407 €$ \\
Berlin & $18237 €$ & Sarre & $13789 €$ \\
Brandebourg & $7330 €$ & Saxe & $1242 €$ \\
Brême & $29159 €$ & Saxe-Anhalt & $9103 €$ \\
Hambourg & $12096 €$ & Schleswig-Holstein & $9624 €$ \\
Hesse & $6728 €$ & Thuringe & $7475 €$ \\
\hline
\end{tabular}

Source des données : Bundesministerium der Finanzen, Auf den Punkt. Bund/Länder Finanzen, 04-08-2014. NB : dette moyenne par tête : $6590 €$.

Règle d'or allemande et Traité TSCG révèlent au grand jour que cette autonomie qui se traduit par une responsabilité budgétaire partielle seulement, et qui a souvent été controversée au fil des décennies mais jamais revue, atteint aujourd'hui ses limites. Ce fait plaide plus que jamais pour que soit attribuée aux Länder une plus large autonomie fiscale, c'est-à-dire en termes de recettes. Elle aurait l'avantage, comme l'explique notamment la Bundesbank dans une étude proposant des orientations pour la réforme du fédéralisme financier, de permettre aux Länder de "mieux... prendre en considération les choix respectifs et de renforcer la perception du lien existant entre impôts et dépenses " (rapport mensuel, septembre 2014). Autrement dit : de les amener à assumer pleinement les conséquences budgétaires de leurs politiques de développement structurel régional et à mieux pondérer leurs stratégies d'investissement public (voir REA 102/2011) en limitant par exemple les dépenses somptuaires (l'exemple souvent cité est celui de l'Elbphilharmonie de Hambourg), ou en maîtrisant mieux les attributions de marchés publics (futur aéroport de Berlin, parc d'attraction du Nürburgring en Rhénanie-Palatinat...).

II est vrai que, en assurant à chaque Land une capacité financière d'au moins $97,5 \%$ de la moyenne, la mise en œuvre concrète du principe de solidarité n'incite guère à la rigueur budgétaire. Du moins en l'état actuel de la pratique : il se manifeste au niveau horizontal sous la forme de la péréquation financière entre les Länder (Länderfinanzausgleich) et, au niveau vertical, sous celle des dotations compensatoires versées par le Bund conformément au principe constitutionnel de "réalisation de conditions de vie équivalentes » sur l'ensemble du territoire (art. 72, 2 de la Loi fondamentale) qui légitime également les aides versées depuis 1995 aux Länder est-allemands au titre du Pacte de solidarité (prolongé en 2005). Les dotations versées aux Länder est-allemands au titre du Solidarpakt II, qui couvre la période 2005-2019, s'élèvent à 156 milliards $€$.

La solidarité inter-étatique au cœur du fédéralisme financier allemand va plus loin encore. Trois cas de figure existent. D'abord celui des tâches communes (Gemeinschaftsaufgaben): une partie des dépenses des Länder est couverte par le Bund dans le cas où ces tâches visent "l'amélioration de la structure économique régionale " (le Bund y contribue alors pour moitié) et "l'amélioration de la structure agricole et de la protection côtière " (le Bund y contribue au moins à hauteur de $50 \%$ ). De même, un co-financement est prévu pour le soutien à la recherche dès lors que son impact dépasse le cadre régional. Ensuite, le cas des mesures adoptées pour parer à une perturbation de l'équilibre économique global, ou pour soutenir la croissance; les aides du Bund sont alors 
limitées dans le temps. Enfin, celui des missions exécutées par les Länder pour le compte du Bund. L'apport du Bund varie selon le type de mission : il est par exemple de $65 \%$ dans le financement du soutien à la formation (BaFöG), de $50 \%$ dans celui de l'aide au logement ou de $100 \%$ dans celui des allocations parentales. Le total de ces dépenses mixtes (Mischfinanzierung) s'élève en 2013 à près de 27 milliards $€$.

Tous ces mécanismes d'interdépendance budgétaire, indispensables lors de la constitution de la République fédérale et de la reconstruction de son économie plurielle, puis après l'Unité pour permettre aux économies est-allemandes de poursuivre leur transition, ont connu au fil du temps une dérive égalitaire. Alors qu'à l'origine, ils reposaient sur le principe d'équité des chances, c'est-à-dire relevaient de l'aide à l'auto-assistance, les transferts massifs qu'ils impliquent ont déployé des effets addictifs et déresponsabilisants, générant au fil du temps une logique de revendication et de transferts de plus en plus indifférenciés, finissant par transformer le principe d'équité en celui d'égalité de droit. Comme chaque Land est assuré au bout du compte d'avoir une capacité financière équivalente à la moyenne, rien ne l'incite à mener sur son territoire une politique de compétitivité accompagnée de réformes visant un gain d'efficience. C'est ce qui avait amené le Tribunal constitutionnel fédéral à formuler en 1999 le principe "d'interdiction du nivellement » (2 BvF 2/98) et à exiger le retour à l'orthodoxie grâce à une réforme de fond du Länderfinanzausgleich.

Or la réforme du fédéralisme financier adoptée en 2010 (et faisant suite à celle du fédéralisme politique de 2006) a éludé ces questions de fond. II est vrai que l'adoption du Pacte de stabilité interne à la RFA (2006) puis de la « règle d'or » (Schuldenbremse ; 2010) avaient alors priorité. En cas de dépassement par l'Allemagne du seuil des $3 \%$ de déficit, le Bund met désormais les Länder à contribution à hauteur de $35 \%$ du total des amendes encourues par l'Allemagne dans I'UE. De plus, à dater du $1^{\mathrm{er}}$ janvier 2016, le déficit structurel du Bund ne peut dépasser $0,35 \%$ du PIB et, à dater du $1^{\text {er }}$ janvier 2020, tout recours au déficit est interdit aux Länder. L'imminence de ces échéances, ajoutée à l'expiration, en 2019, de la loi sur le Pacte de Solidarité II et de celle sur les critères de distribution des recettes fiscales entre les Länder, remet à l'ordre du jour ces questions de fond. Et fait apparaître dans le même temps le potentiel de compétitivité bridé jusqu'ici par un système de redistribution qui a perdu toute justification et toute transparence au fil du temps.

Car aujourd'hui, ces mécanismes de solidarité non seulement ne 'profitent' à aucun Land, pénalisant les plus compétitifs, les empêchant d'accroître encore leurs performances, tout en freinant l'effort des moins compétitifs pour qui ils s'assimilent à une "véritable trappe à pauvreté ", comme le formule dans une étude l'Institut der deutschen Wirtschaft (IW ; 2014). Plus généralement, pour l'Allemagne dans son entier, cette redistribution généralisée rend difficile la poursuite des réformes structurelles et tasse la croissance potentielle du PIB. Le jugement du Conseil des Sages est dès lors sans appel : le système actuel est « hautement inefficace » (rapport annuel 2014).

\section{Enjeux et principaux axes de réforme actuellement débattus}

Avec toutes ses interdépendances et les multiples et complexes critères de redistribution qui lui sont propres (nous n'en avons esquissé ici que les principaux), mais aussi les liens avec les questions plus générales que sont le financement des infrastructures, le code fiscal ou la répartition des compétences au sein du fédéralisme politique, le dossier du fédéralisme financier est d'une extrême technicité dans le détail. Au point que les négociations de réforme en cours s'apparentent à un gigantesque marchandage dont le pivot est la question des contreparties en échange d'abandons de souveraineté et de participation à la redistribution générale de l'encours fiscal. De surcroît, comme dans la phase actuelle de négociation de grandes lignes directrices, les réflexions qui
Dérive égalitaire

Pacte de stabilité interne et « règle d'or " révèlent l'urgence d'une réforme

La solidarité s'est transmuée en trappe à pauvreté

Entrelacs d'enjeux 
Des négociations non publiques, mais malgré tout accessibles

Tractations et monnaies d'échange

En attente d'un arrêt de Karlsruhe portent sur la révision des flux de transferts à la fois horizontaux et verticaux sont menées au niveau de l'exécutif seul (groupes de travail des ministres des Finances des Länder, ou bien des 16 ministres-présidents, ou bien des ministres-présidents SPD seulement, sommets à périmètre variable incluant le gouvernement fédéral...), le Bundestag n'est pas encore impliqué. II le sera lorsque les projets de loi afférents seront rédigés et soumis au vote parlementaire pour adoption par le Bundestag (la chambre des députés) et le Bundesrat (la représentation de l'exécutif des Länder à l'échelon fédéral). Or les parlements des Länder exigent d'être impliqués eux aussi ; après tout, ce sont eux qui votent le budget de leur Land...

Mais, dans la démocratie allemande où le citoyen a un droit à l'information - a fortiori lorsqu'il s'agit de choix politiques importants et de ses impôts -, la presse relate abondamment les négociations en cours. D'autant que la réforme du fédéralisme financier est le chantier de réforme majeur du gouvernement de coalition et doit être achevée impérativement en 2017, ce qui suffit à placer ce sujet en tête de l'agenda. Le ministère fédéral des Finances, quant à lui, publie sur son site une brochure grand public expliquant l'enjeu de la réforme à venir : jeter les bases de l'avenir de l'Allemagne dans "la poursuite de l'européanisation et la globalisation ». Les Allemands sont donc bien informés, d'autant que la problématique comme les enjeux sont connus depuis longtemps. A intervalles réguliers en effet, le Conseil des Sages, les instituts économiques, le patronat et le mouvement syndical, des think tanks indépendants (par ex. Konvent für Deutschland) ou encore la Bundesbank, publient des préconisations de réforme, sans parler de la Cour de Karlsruhe qui a eu à connaître de nombreuses plaintes en constitutionalité sur divers aspects de ce partage des ressources. Quoi qu'il en soit, lors du sommet réunissant le 11 décembre les ministres des Finances des Länder, leur homologue fédéral et la chancelière, un premier accord doit être trouvé. En théorie du moins, car rien n'est moins sûr.

Dans les tractations en cours, où s'affrontent les échelons territoriaux (communes comprises), mais aussi les appartenances politiques des ministres-présidents (chefs de gouvernement des Länder), différentes questions s'entremêlent : refonte de la péréquation horizontale entre les Länder, révision des dotations compensatoires versées par le Bund aux Länder, institution de programmes de réformes et de consolidation des finances publiques supervisés par le Conseil de stabilité interne à l'Allemagne, création d'un fonds fédéral pour apurer tout ou partie les comptes des Länder les plus endettés, création de "Deutschland-Bonds », avenir de la contribution de solidarité, élargissement de l'autonomie fiscale des Länder, répartition différente du produit des impôts communs, réforme de la fiscalité des ménages, allégement des charges financières des Länder par une révision des missions qu'ils exercent pour le compte de la Fédération... Et même, bien que furtivement, resurgit la question d'une éventuelle fusion de certains Länder... Toutes ces questions servent ainsi de monnaie d'échange les unes aux autres dans ces négociations en cercles concentriques: Bund et Länder, Länder entre eux, Länder de l'est à part, Länder au gouvernement SPD/Verts entre eux, Länder et communes, communes...

Et sur ces débats pèse une épée de Damoclès : un arrêt à venir (en 2015 ?) de la Cour de Karlsruhe. La Bavière et la Hesse, deux des principaux contributeurs dans le cadre de la péréquation horizontale entre les Länder (étage 3 du fédéralisme financier), avaient déposé, le 23 mars 2013, une plainte en constitutionnalité auprès de la Cour de Karlsruhe afin de rétablir l'équité de l'organisation actuelle de ce système de solidarité financière et de le rendre à l'avenir plus incitatif en termes de compétitivité économique. "Un acte de légitime défense politique » pour Horst Seehofer (CSU), ministre-président de Bavière. Les premières négociations engagées entre les Länder dès le début de la législature pour réformer le Länderfinanzausgleich et réduire la contribution des trois Länder du sud avaient en effet échoué face au refus obstiné des bénéficiaires, menés par Hannelore Kraft (SPD), ministre-présidente de la Rhénanie du Nord- 
Westphalie (Land en concurrence frontale avec la Bavière). II y a près de vingt ans déjà, dans un contexte similaire, la Bavière, la Hesse, ainsi que le BadeWurtemberg avaient saisi à ce propos la Cour de Karlsruhe, à la suite de quoi les Juges suprêmes avaient rappelé le principe de l'interdiction du nivellement et avaient fait injonction aux gouvernements de réformer le Länderfinanzausgleich. Il en était sorti une "réformette » (voir Gabel, 2001), faute de volonté partagée d'aborder les questions de fond. Mais comme souvent, la simple perspective d'une possible invalidation des modalités actuelles par la Cour de Karlsruhe pourrait aujourd'hui forcer le compromis malgré des positions très tranchées et en apparence inconciliables - notamment sur le cas de Berlin, qui absorbe l'essentiel des fonds répartis horizontalement entre les Länder sans parvenir à gagner en compétitivité ni à réduire sa dette pour autant.

Contributeurs et bénéficiaires campent actuellement d'autant plus fermement sur leurs positions que les premiers, ainsi que la très vertueuse Saxe, ont des ministres-présidents CDU/CSU (sauf le Bade-Wurtemberg dont le chef de gouvernement est Vert, ce qui explique en partie qu'il ne se soit pas joint aux plaignants) et que les seconds sont majoritairement gouvernés par le SPD seul ou une coalition SPD/Verts - dont Berlin, Brême ou la puissante Rhénanie du Nord-Westphalie. Ces derniers, également plus lourdement endettés que les contributeurs, ont dès lors formulé une proposition jugée inacceptable par les trois 'riches' Länder du sud : pérenniser le Solidaritätszuschlag, en faire un impôt commun et l'intégrer en 2020 dans l'IR, ce qui accroîtrait d'autant le volume de la part qui leur revient dans le partage des impôts communs (42,5\%), ainsi qu'aux communes sur leur territoire (15\%). La Bavière et la Hesse refusent une telle solution qui, selon eux, s'assimilerait à une hausse de l'impôt sur le revenu. Or il est question aussi, au cours de la législature, de réformer le code fiscal pour notamment réduire la charge de l'IR.

Le Solidaritätszuschlag (surnommé "Soli »), cette contribution de solidarité prélevée depuis 1992 sur l'IR et l'IS dans le cadre de l'Aufbau Ost pour financer entre autres l'effort lié à la réunification, est un impôt direct (barème : $5,5 \%$ depuis 1998) qui revient en propre au Bund. Son produit est de l'ordre de 15 milliards $€$ en 2014 et pourrait atteindre 18 milliards $€$ à la fin de la décennie. Conçu à l'origine comme un prélèvement transitoire pour accompagner l'Unité allemande et affecté aux Länder de l'est, le produit de cet impôt est aujourd'hui versé au budget général du Bund ; il a notamment permis de financer l'intervention en Irak. Pour Wolfgang Schäuble (CDU) non plus, la solution proposée par les Länder SPD et SPD/Verts, Rhénanie du Nord-Westphalie en tête (le Land pilote le groupe des Länder SPD), n'est donc pas envisageable, du moins sans contrepartie, par exemple une révision de la clé de partage de la TVA. En effet, à l'occasion de l'intégration des nouveaux Länder dans le système du Länderfinanzausgleich, en 1995, le Bund avait cédé aux Länder, en contrepartie, pas moins de sept points de pourcentage de sa part de TVA. Pourtant, sous l'angle du droit constitutionnel, comme il s'agit d'un prélèvement ad hoc, son existence perd toute justification après l'expiration du Solidarpakt II, qui scellera la fin d'un besoin de financement spécifique pour les Länder issus de l'ex-RDA, comme le rappelle Hans-Jürgen Papier, ancien président du Tribunal constitutionnel fédéral. En effet, la Constitution "interdit d'utiliser un prélèvement d'appoint pour une hausse durable de la fiscalité » (Die Welt, 05-08-2013). Et nombre d'experts, mais aussi la Commission parlementaire CDU, plaident pour qu'il expire en 2019, de même que la fédération des contribuables ou les milieux patronaux (essentiellement le Mittelstand). Quant à la Cour de Karlsruhe, elle a été saisie (renvoi) en août 2013 pour contrôle en constitutionnalité par la Cour financière du Land de Basse-Saxe.

Le gouvernement fédéral souhaiterait au contraire inscrire cet impôt dans la durée. Certes, la chancelière, qui redoute une hausse de l'IR induite, refuse l'idée de la transformation du «Soli » en impôt commun. Mais le ministre fédéral des Finances pourrait l'envisager, à condition que les Länder cèdent sur certaines
Opposition Union/SPD sur l'avenir du Solidaritätszuschlag

Sa pérennisation pose un problème de droit constitutionnel

Affecter le «Soli » à la réduction de la dette des Länder? ... 
... et surtout des communes, entités les plus endettées?

Institution d'un fonds commun d'amortissement de la dette? prérogatives au sein du Länderfinanzausgleich et se montrent prêts à prendre à leur charge une partie du service de la dette des plus endettés d'entre eux (et de leurs communes). Cette proposition avait été élaborée conjointement avec Olaf Scholz (SPD), maire et chef de gouvernement de Hambourg, dans le cadre de négociations parallèles. Mais elle semble peu réalisable dans la mesure où elle signifierait une hausse de la contribution de la Bavière, du Bade-Wurtemberg et de la Hesse dans le cadre du Länderfinanzausgleich. Une autre solution, envisagée un temps conjointement avec les communes, pourrait être celleci : le «Soli » resterait un impôt fédéral, mais le Bund, en contrepartie, prendrait en charge l'intégralité du financement des aides au chauffage et au logement destinées aux chômeurs (régime Hartz IV et actuellement à la charge des communes (déjà, sous l'actuelle grande coalition, il contribue à hauteur de quelque 14 milliards $€$ au financement de l'aide à l'insertion des handicapés, qui relève des communes). Mais cela réduirait d'autant la responsabilité, déjà amenuisée, des communes pour la gestion des aides attribuées au titre de Hartz IV et ne les inciterait pas à plus de rigueur budgétaire. Or une telle solution s'inscrit dans un autre axe de réflexion encore, celui d'un nouveau partage des compétences entre Bund et Länder dans le domaine social : les Länder pourraient se voir confier un pouvoir législatif partiel en ce qui concerne les prestations sociales qu'ils financent, avec pour résultat une différenciation des standards au sein de l'Allemagne. C'est là aussi, soit dit en passant, une pique à destination de Berlin dont la dette a pour origine notamment " une pratique plus généreuse des transferts » sociaux, comme le formulait la Cour de Karlsruhe dans un arrêt rendu en 2006 et où il déboutait le Land de Berlin qui exigeait l'aide du Bund pour surmonter sa "crise budgétaire extrême » (voir REA 79/2006). Quoi qu'il en soit, dans une libre opinion parue le 20-09-2014 dans le quotidien F.A.Z., W. Schäuble rappelle que « la marge de manœuvre du Bund est limitée ».

"Limitée » mais pas nulle. Le dossier reste donc ouvert, quelle que soit la forme que pourrait prendre la pérennisation du Solidaritätszuschlag - à condition toutefois que la Cour de Karlsruhe considère qu'elle est conforme à la Loi fondamentale. Une telle manne potentielle est idéale comme monnaie d'échange dans les négociations, notamment avec les Länder SPD/Verts. Elle suscite de vives convoitises depuis longtemps - à l'ouest, et principalement en Rhénanie du Nord-Westphalie. Elle pourrait servir à moderniser les infrastructures de transport. Mais elle serait surtout idéale pour boucher les trous budgétaires des communes et amortir leur dette. Car avec l'entrée en vigueur de la « règle d'or » pour les Länder au $1^{\mathrm{er}}$ janvier 2020, ce sont eux qui devront assumer la dette de leurs communes. Or ces dernières sont lourdement endettées : leur dette s'élève à 82,9 milliards $€$ au total, soit $1108 €$ par habitant en moyenne en 2013. A cela s'ajoutent des crédits à court terme d'un montant total de 48,6 milliards $€$ (ministère fédéral des Finances). C'est à l'ouest que les communes sont le plus endettées, avec $1165 €$ par habitant contre $825 €$ à l'est. Et depuis longtemps, celles de l'ouest réclament un «Soli ouest », ou du moins une dotation spécifique calculée en fonction du degré de nécessité - surtout celles qui doivent affronter la restructuration, comme c'est le cas en Rhénanie du Nord-Westphalie. Quoi qu'il en soit, 59 communes de ce Land s'apprêtent à saisir la Cour de Karlsruhe contre la péréquation horizontale inter-communale actuelle de leurs recettes fiscales, qui fait obligation aux plus riches d'entre elles de reverser une partie de leur encours aux plus déficitaires. Plus généralement, les fédérations des communes verraient d'un bon œil l'abolition de l'ancien régime et son remplacement par l'affectation du produit d'un «Soli » redéfini en dotation de solidarité soumise à conditions de nécessité. Les partis politiques abondent tous dans le même sens. Reste à savoir quelle forme pourrait prendre cette dotation, quelle en serait la contrepartie et quel échelon devrait en assurer le financement.

Cette situation soulève aussi plus généralement la question de la manière dont il convient de gérer la dette cumulée des Länder et des communes. Rappelons 
que l'objectif de la Grande coalition est de ramener la dette à $70 \%$ du PIB en 2017, ce qui exige des efforts conséquents de chaque entité territoriale, le niveau de la dette allemande étant actuellement de 76,9\% (Eurostat). Dans ce contexte, l'encours du «Soli » pourrait alimenter un fonds d'amortissement de la dette des Länder et communes : la charge des intérêts serait supportée par le Bund, et les Länder disposeraient de 50 ans pour rembourser leur dette. Cette solution alternative à la transformation en impôt commun, et proposée par Olaf Scholz, fait l'unanimité dans le camp des Länder SPD/Verts. Les 18 milliards $€$ que produira le «Soli » à la fin de la décennie permettraient en effet de couvrir la charge des intérêts. Constitutionnellement aussi, une telle idée est envisageable, puisqu'il revient au Bund de veiller à "l'amélioration de la structure économique régionale ". Mais ce projet se heurte à la farouche opposition des Länder CDU/CSU qui invoquent la clause implicite de no bail-out qui prévaut dans le fédéralisme financier allemand. "Le Soli n'a pas à être un libre service pour les Länder SPD », s'insurge par exemple Markus Söder (CSU), ministre des Finances de Bavière (Mittelbayerische Zeitung, 24-06-2014) ; quant à Thomas Schäfer (CDU), son homologue de Hesse, il précise : "Un fonds commun d'amortissement de la dette favoriserait ceux qui ont accumulé une dette disproportionnée »(Die Welt, 24-06-2014). II s'agit là de gesticulations habituelles dans le cadre des discussions sur le fédéralisme financier allemand. En réalité, les négociations sur ce point pourraient très bien aboutir à un compromis, comme le laisse entendre Annegret Kramp-Karrenbauer (CDU), ministreprésidente de la Sarre, un Land lourdement endetté (voir REA 114/2014) : « La proposition de M. Scholz mérite réflexion. Le Soli présente une opportunité pour aborder la question des dettes » (Die Welt, 24-06-2014). Quant au ministère fédéral des Finances, il affiche son opposition, invoquant une marge de manœuvre budgétaire du Bund affaiblie notamment par l'apport de l'Allemagne au MES. Et aussi par la hausse prévisible des dépenses sociales du Bund, à quoi les Länder rétorquent qu'ils verront, eux, exploser leur poste affecté aux pensions de retraites des personnels du public.

Certes, deux doctrines s'affrontent au sein de l'Allemagne sur le recours à l'endettement, ce qui incite Wolfgang Schäuble à rappeler que «tout incite à penser que presque tous les Länder seront capables de respecter la règle d'or d'ici 2020 sans difficulté insurmontable. La seule condition est qu'ils mènent une politique budgétaire rigoureuse - elle est le fondement même d'une attitude coopérative au sein d'un Etat fédéral » (F.A.Z., 20-06-2014). Mais ce ne sont là que des débats de façade ; le compromis est à portée de main. En contrepartie de l'institution d'un fonds commun d'amortissement de la dette des Länder (et communes), le Conseil de Stabilité (Stabilitätsrat) interne à l'Allemagne pourrait voir ses pouvoirs renforcés et être habilité à prononcer des sanctions à l'encontre des Länder refusant de se plier à la discipline budgétaire. Cette instance de contrôle budgétaire, composée du ministre fédéral des Finances, de ses homologues des Länder et du ministre fédéral de l'Economie, avait été instituée en avril 2010 dans le cadre de l'adoption de la "règle d'or " (voir REA 102/2011). Elle a pour mission de contrôler les budgets annuels du Bund et des Länder pour prévenir les risques de " situation budgétaire extrême » et engager les mesures appropriées d'assainissement de leurs finances publiques.

Cette extension des missions préventives du Conseil de Stabilité à des pouvoirs de sanction en cas de déficit excessif, dont celui de saisir le Tribunal constitutionnel fédéral pour non-respect des principes au fondement de cette «communauté solidaire » qu'est la République fédérale, donnerait plus de consistance au Pacte de Stabilité interne à la RFA et le rendrait plus contraignant. Mais ce projet est vivement critiqué par la plupart des Länder qui y voient une tentative de mainmise du Bund sur le contrôle de leurs finances, donc une perte de souveraineté. Mais les esprits pourraient être pacifiés. Car dans le cadre de ces réflexions est débattu également un assouplissement de la « règle d'or »: les $0,35 \%$ du PIB de déficit structurel auxquels a droit le Bund à partir de 2016
Vers des pouvoirs renforcés pour le Conseil de Stabilité

Contrepartie :

assouplissement de la « règle d'or » pour les Länder? 
Mutualisation de la dette des Länder?

Réforme de fond du Länderfinanzausgleich?

Incongruité de la pondération du nombre d'habitants pourraient donner lieu à partage avec les Länder, le Bund conservant une marge de 0,2\% et attribuant les 0,15 \% restants aux Länder. Ils n'auraient donc plus à se plier à la règle du $0 \%$ en 2020 . Seulement, il faudrait pour cela modifier la Constitution.

Une autre question encore est soumise à réflexion dans le contexte de l'institution d'un fonds d'amortissement: la création de Deutschland-Bonds. Comme c'est le Bund qui prendrait à sa charge également le service de la dette des Länder (et de leurs communes), il serait logique que l'émission d'obligations d'Etat soit mutualisée, avancent Wolfgang Schäuble et Olaf Scholz (du fait de son indépendance budgétaire, chaque Land émet actuellement ses propres obligations). Cela présenterait l'avantage de lisser le spread interne à la RFA et de permettre aux Länder moins bien notés de profiter de taux d'intérêts avantageux, puisqu'ils bénéficieraient de l'excellente notation de la RFA sur les marchés. Mais cette idée soulève un tollé : la Bavière, le Bade-Wurtemberg, la Saxe (et la Thuringe sous le précédent gouvernement) y sont farouchement opposés, estimant qu'une telle mesure aurait des effets néfastes puisqu'elle inciterait les Länder les plus lourdement endettés à relâcher leurs efforts de rigueur budgétaire. Déjà, les mêmes avaient refusé de participer à une première tentative d'émission de Deutschland-Bonds à l'été 2013 ; elle s'était révélée un semi-échec, faute de participation des Länder les plus compétitifs et les plus solvables. Au sein de l'Allemagne, les arguments pour et contre sont donc les mêmes qu'à l'échelon de la zone Euro en ce qui concerne les Eurobonds... Mutualisation, oui, mais seulement lorsque tous les Etats auront fait la preuve qu'ils assument leur responsabilité collective.

Sur tous ces dossiers relatifs à la répartition verticale des ressources, les axes verticaux et horizontaux sont étroitement entremêlés. II en va de même de la négociation de l'autre grand volet de la réforme du fédéralisme financier : celle du Länderfinanzausgleich - la péréquation horizontale entre les Länder, qui fait l'objet depuis plusieurs décennies de tentatives de réforme, toujours ajournées quant au fond. Mais cette fois, dans le contexte du chantier de la réforme majeure du fédéralisme allemand, la troisième ("Föderalismusreform III ») après celles du fédéralisme politique en 2006 et du fédéralisme budgétaire en 2010, les questions de fond sont abordées. Les contraintes du Pacte de stabilité interne à la RFA comme du TSCG ne laissent plus d'autre choix. Ni non plus l'injonction faite par la Cour de Karlsruhe. Dans son arrêt du 11-11-1999, elle avait fixé aux Länder un calendrier de réforme contraignant : redéfinir les critères de répartition des ressources avant 2003 et adopter avant la fin 2005 une loi sur les modalités concrètes de cette répartition. Or les Länder ne l'avaient que partiellement respecté, adoptant certes la loi exigée (en 2001), mais reportant aux calendes grecques la révision de fond en comble de ces critères de partage (voir REA 52-53, 2001). Ils s'étaient contentés d'un ajustement ; or la loi afférente (Maßstäbegesetz), en vigueur depuis 2005, expire à la fin 2019. Sa révision est donc doublement à l'ordre du jour, puisqu'aujourd'hui les mêmes plaignants qu'en 1999 - Bavière et Bade-Wurtemberg (la Hesse en faisait également partie à l'époque) - ont saisi à nouveau le Tribunal constitutionnel fédéral sur la même problématique du nivellement, incriminant cette fois l'injustice foncière de certains critères de répartition.

Le problème qu'ils soulèvent (et qu'incriminent également la Bundesbank, le Conseil des Sages et la plupart des scientifiques) est simple: l'incongruité totale de la pondération du nombre d'habitants pour le partage de l'encours fiscal et la redistribution horizontale. Cette pondération varie en effet, les critères déterminants n'étant pas les mêmes selon qu'il s'agit d'affecter les $25 \%$ de retenue sur le produit de la TVA, de calculer la part des autres impôts revenant au Land ou aux communes. En matière de TVA, le critère est le nombre réel d'habitants, dans tous les autres cas, celui de leur pondération, et encore selon des clés variant en fonction soit du type de Land (les villes-Etat que sont Berlin, Brême et Hambourg ont un statut particulier), soit de la comptabilisation dans le 
budget du Land ou non d'une part des recettes fiscales de ses communes. Concrètement, c'est le lieu de perception qui prévaut pour l'IR, en l'occurrence le lieu de résidence (et non le lieu de travail, ce qui ne permet pas de tenir compte des phénomènes migratoires). Pour l'IS comme pour la taxe professionnelle communale, c'est le lieu d'implantation de l'entreprise ou du site de production (part de la masse salariale répartie selon les sites). La TVA, enfin, est répartie en deux temps. D'abord, sur le total revenant aux Länder, $25 \%$ sont prélevés pour amener la capacité financière de chaque Land à $94,5 \%$ de la moyenne, la clé de répartition étant la suivante : le produit par habitant est corrigé des différences constatées entre les Länder en ce qui concerne tant le niveau des recettes fiscales du Land que la moyenne des barèmes en vigueur dans le Land. Dans cette première étape sont redistribués quelque 7,5 mil liards $€$, rappelle la Bundesbank (Rapport de septembre 2014). Ensuite, la répartition des $75 \%$ restants suit le schéma (simplifié) suivant : pour calculer la part revenant à un Land, on tient compte des recettes fiscales d'un Land (définies en tant que besoins budgétaires), en y incluant $64 \%$ des recettes de ses communes, mais corrigées des différences des barèmes appliqués à la taxe professionnelle et à la taxe foncière. Les besoins budgétaires correspondent à ceux estimés nécessaires à un Land pour pouvoir remplir ses missions dans le partage des tâches sectorielles (législatives) et fonctionnelles (pouvoir administratif) qui caractérise le fédéralisme allemand.

La péréquation horizontale entre Länder postule l'égalité des besoins financiers par tête de tous les habitants de la RFA. Or la compétitivité, la structure des activités, et même la taille des Länder variant grandement, deux régimes dérogatoires sont prévus: l'un concerne les Länder à faible densité de population (Brandebourg, Mecklembourg et Saxe-Anhalt), l'autre les trois villes-Etats que sont Berlin, Brême et Hambourg. Ces Länder bénéficient donc d'une pondération spécifique de leur nombre d'habitants ("Einwohnerveredelung »). La plus problématique sous l'angle de l'équité est celle qui s'applique aux villes-Etats : elles bénéficient d'une majoration de $35 \%$ (tous types d'impôts communs), destinée à couvrir les frais particuliers liés à leur statut de métropole et à leur rayonnement dans les Länder environnants. C'est ce régime qui est au cœur de la plainte en constitutionnalité déposée par la Bavière et le Bade-Wurtemberg. Car ces trois villes-Etats étant également des communes, elles cumulent les impôts propres à ces deux entités ; la justification d'un traitement particulier ne s'impose donc pas à l'évidence, puisqu'ainsi " la capacité financière d'une villeEtat est par nature supérieure ", explique Markus Söder, ministre des Finances de Bavière (Deutschlandfunk, 21-09-2014). Supprimer cette pondération spécifique permettrait de rétablir une certaine équité de traitement et accessoirement de réduire la différence de traitement entre Länder de l'est et de l'ouest; elle ne se justifie plus guère, les premiers ayant pratiquement achevé leur transition.

Reste à trouver une solution pour le cas très particulier de la ville-Etat de Berlin qui se trouve en situation budgétaire " tendue ». Le Land avait saisi en 2003 la Cour de Karlsruhe pour arbitrer le contentieux qui l'oppose depuis longtemps au Bund. II estimait avoir droit aux mêmes aides fédérales que les Länder de Brême et de Sarre à qui le Bund les avait accordées en 1992 (à la suite d'un précédent arrêt de Karlsruhe). Or la Cour a débouté Berlin, estimant que, comme les efforts de consolidation menés entre 1995 et 2004 n'avaient pas réduit significativement les dépenses de fonctionnement et les transferts sociaux, les problèmes budgétaires de Berlin "se situent plutôt du côté des dépenses que de celui des recettes", ajoutant que son "potentiel d'économies est loin d'être épuisé » (voir REA 79/2006). Or, le programme d'assainissement de ses finances publiques élaboré depuis au sein du Stabilitätsrat n'ayant toujours pas porté de fruits, une solution devient d'autant plus urgente à trouver que le soutien démesuré que lui apportent les trois länder donateurs (Bavière, BadeWurtemberg et Hesse) via le Länderfinanzausgleich ( $40 \%$ du total des transferts) est tout sauf équitable en comparaison des autres Länder et qu'il s'est 
Fusion des Länder? C'est là que resurgit une autre proposition, débattue à de nombreuses reprises par le passé et toujours rejetée : une fusion de certains Länder. Dans un long entretien accordé au quotidien Die Welt (05-08-2013), Hans-Jürgen Papier, ancien président du Tribunal constitutionnel fédéral évoquait : "Nous devrions réfléchir à une réorganisation territoriale de la RFA ". La Bundesbank aussi, au détour d'une phrase de son rapport mensuel de septembre 2014, évoque ce point. Et un an plus tard, Annegret Kramp-Karrenbauer, ministre-présidente (CDU) de la Sarre, un Land lourdement endetté et bénéficiaire d'un soutien ad hoc du Bund (voir REA 114//2014), relance cette réflexion dans les négociations avec ses homologues : "Il faut que nous demandions s'il ne devrait pas y avoir à l'avenir seulement six ou huit Länder au lieu des 16 actuels " (Süddeutsche Zeitung, 24-10-2014). Or cette proposition, bien que réalisable puisque la Loi fondamentale prévoit la possibilité d'une telle réorganisation, ne semble guère retenir leur attention, ni celle du Bund, tant elle touche aux fondements mêmes de l'identité fédérale de l'Allemagne. Et aussi, il faut bien le dire, tant elle est malaimée par les électeurs. La dernière tentative, le référendum de 1996 sur la fusion entre Berlin et Brandebourg, qui avait pourtant du sens puisque les deux territoires ne font économiquement qu'un, avait lamentablement échoué. Et, diversité des identités culturelles oblige, il n'est pas certain que les Sarrois aient envie de fusionner avec leurs voisins de Rhénanie-Palatinat, ni les Brêmois avec les Bas-Saxons, ni les Hambourgeois avec leurs voisins ou de Basse-Saxe ou du Schleswig-Holstein, ni non plus les Saxons avec les Thuringeois et leurs voisins de Saxe-Anhalt. Pourtant, une telle option permettrait de rendre caduc le Länderfinanzausgleich, de résoudre en partie le problème de la compétitivité et de la dette souveraine des Länder respectifs. De fait, Annegret Kramp-Karrenbeuer n'avait lancé cette suggestion que pour tenter d'accélérer les négociations sur la gestion de la dette des Länder. Mais elle a pris soin aussi de préciser que ce n'était pas la justification prioritaire d'un redécoupage territorial.

Vers plus de Reste la question des recettes des Länder. L'un des axes clés du débat de concurrence fiscale? réforme porte ainsi sur la question de savoir comment et dans quelle mesure accroître l'autonomie fiscale des Länder. En leur permettant de prélever partiellement ou totalement des impôts en propre, elle leur donnerait une meilleure maîtrise de leurs budgets, leur faciliterait consolidation budgétaire et remboursement de leur dette, c'est-à-dire qu'elle leur donnerait la pleine ampleur des moyens pour exercer leurs compétences constitutionnelles et pour optimiser leurs politiques de stabilité et de croissance. II s'agirait principalement, soit de conférer aux Länder le droit de fixer une part variable des impôts communs (IR et IS) qui leur reviendrait en propre, soit de la rétrocession d'une partie de la souveraineté fiscale fédérale aux Länder, comme le proposent régulièrement et le Conseil des Sages et la Bundesbank, et comme ils viennent de le faire à nouveau respectivement dans le rapport mensuel de septembre et dans le rapport annuel de novembre 2014. Si les Länder CDU/CSU saluent la dose de concurrence inter-étatique qu'introduiraient de telles options, les Länder à dominante 
SPD s'opposent à un « dumping fiscal » interne à l'Allemagne. Néanmoins, tous verraient d'un bon œil une plus large latitude d'action. Markus Söder (CSU) résume ainsi les positions respectives : "De la sorte, les uns pourraient légèrement augmenter leurs impôts - ce qu'ils souhaitent au demeurant -, et les autres à l'inverse les baisser » (Deutschlandfunk, 21-09-2014). Quant au Bund, il serait favorable à un peu plus de concurrence.

Cette question est délicate à résoudre puisqu'elle se heurte à la doctrine au fondement du fédéralisme coopératif allemand. Dès lors, le Conseil des Sages, prenant pour exemple le modèle du fédéralisme suisse (comme la Bundesbank d'ailleurs), prend soin de préciser: "Une telle autonomie renforcée pourrait être aménagée de manière à éviter que s'installe une concurrence fiscale ruineuse ou une divergence entre les Länder à forte et à faible capacité financière. Le renforcement de l'autonomie en matière de recettes doit donc se concevoir comme une solution complémentaire à la péréquation financière solidaire, et non se substituer à elle ". II s'agit donc de réviser le mode de fonctionnement du fédéralisme financier tout en pérennisant le principe de solidarité sur lequel il repose. Ce qui plaide, comme le formule le Conseil des Sages dans l'intitulé du chapitre qu'il consacre aux préconisations de réforme, pour " une Constitution financière activante » ("Für eine aktivierende Finanzverfassung »).

"NOMBREUSES SONT LES PROPOSITIONS faites dans les négociations en cours. Mais la plupart d'entre elles consistent jusqu'ici en la revendication de plus de moyens formulée par les Länder, et non en propositions montrant la volonté de donner corps à une autre conception. Or ce n'est que si les Länder apportent une contribution véritable aux réflexions sur la manière de rendre plus efficient et plus compétitif l'Etat dans son ensemble que l'Etat fédéral [qu'est la RFA] restera à l'avenir en pleine possession de ses moyens et de sa capacité d'agir ». C'est ainsi que Wolfgang Schäuble conclut sa tribune libre publiée le 20 septembre dernier dans le quotidien F.A.Z. Pour l'instant, les négociations, souvent mues par des égoïsmes exacerbés, portent sur d'innombrables points de détail, certes importants, mais elles semblent avoir perdu de vue l'enjeu foncier que représente la réforme en cours et qui doit être achevée en 2017. II est vrai qu'il tient de la quadrature du cercle : veiller à l'homogénéité des conditions de vie sur tout le territoire sans aboutir à un nivellement en termes de compétitivité comme de capacité financière, assurer à chaque entité les moyens de mettre en œuvre ses missions sans pénaliser les plus performants...

Par-delà le cas particulier de l'Allemagne et de son effort de modernisation de ses complexes rouages budgétaires pour "créer des conditions favorables aux investissements d'avenir " grâce à des finances publiques solides, comme le formule le point 3 du Contrat de coalition Union/SPD, il s'agit ni plus ni moins d'apporter collectivement, à tous les échelons territoriaux, "une contribution à la stabilité de la zone Euro ", poursuit ce contrat. En effet, "nous sommes conscients de la responsabilité qui incombe à l'Allemagne de tenir son rôle en Europe en menant une politique financière et budgétaire rigoureuse et soutenable ". C'est exactement pour la même raison que la Bundesbank plaide "pour que la supervision des budgets publics au sein de l'Etat fédéral allemand prenne une forme plus sévère qu'à l'échelon européen ". Indépendamment des épineuses questions de redistribution interne des richesses, c'est une nouvelle articulation entre solidarité et responsabilité que l'Allemagne doit trouver - pour elle-même.

Sous couvert de solidarité, l'Allemagne fédérale est devenue au fil du temps une union de transferts. Elle a fait l'expérience du nivellement auquel peut mener une solidarité sans contrepartie, de même qu'une souveraineté budgétaire sans la contrainte de la responsabilité collective. Elle connaît aussi les effets pervers, déresponsabilisants - le cas de Berlin en atteste, mais il est loin d'être le seul - d'une aide exceptionnelle accordée d'en haut (par le Bund en l'occurrence), d'abord ultima ratio puis définitivement, à un Land en difficulté. L'enjeu 
de cette réforme est bien sûr d'asseoir la compétitivité de l'Allemagne sur des bases financières plus saines. Et pour cela, il faut moderniser l'organisation concrète du fédéralisme adoptée voici quarante ans. Cette modernisation est aussi un signal fort donné aux partenaires dans la zone Euro et dans le reste de I'UE. Mais les réformes de structure ne sont jamais simples, en Allemagne non plus...

\section{Indications bibliographiques}

- Bourgeols I. (dir), Allemagne: compétitivité des territoires, Coll. Travaux et Documents du CIRAC, Cergy-Pontoise, 2007. Voir en particulier: BouRGEOIS I., "La gouvernance territoriale allemande à l'épreuve de la globalisation et de l'intégration européenne » et "Compétitivité des territoires : état des lieux et perspectives ", ainsi que : GABEL M., "Aufbau Ost : entre rattrapage et différenciation »

- BourgeoIs I., "Fédéralisme financier. Solidarité financière - l'expérience allemande ", Regards sur l'économie allemande, $\mathrm{n}^{\circ} 102$, automne 2011, et « Pas d'exception budgétai re pour Berlin », Regards sur l'économie allemande, $\mathrm{n}^{\circ} 79$, décembre 2006

- BouRgeoIS I., LASSERRE R., Les facteurs systémiques de la compétitivité allemande, avril 2011, www.cirac.u-cergy.fr/colloques etudes_wp/CIRAC_CNI.pdf

- BRÜGELMANN R., "Föderalismusreform: Die Unzulänglichkeiten des Finanzausgleichs unter den Ländern », IW Policy paper, 16/2014

- Brügelmann R., Tröger M., "Die deutsche Finanzverfassung ", in INSTITUt DeR DeUtSchen WIRTSCHAFT KöLn (ed), Föderalismus in Deutschland. Ökonomische Analyse und Reformbedarf, Cologne, 2007

- BUNDESMinisterium DeR Finanzen, Bund/Länder-Finanzbeziehungen auf der Grundlage der Finanzverfassung, éd. 2014, Bund/Länder Finanzen, Auf den Punkt, 04-08-2014, "Ergebnisse des Länderfinanzausgleichs 2013 ", Monatsbericht, février 2014

- DeUtSChe BundesbanK, «Zur Reform der föderalen Finanzbeziehungen », Monatsbericht, septembre 2014, et « Defizitbegrenzende Haushaltsregeln und nationaler Stabilitätspakt in Deutschland », Monatsbericht, avril 2005

- Freigang D., Ragnitz J., "Die Ergebnisse der Föderalismusreformkommission II und die Bewertung der Verschuldungsgrenze ", ifo Dresden berichtet, 3/2009

- Gabel M., "La réforme du fédéralisme financier allemand. Beaucoup de bruit pour rien ?", Regards sur l'économie allemande, $n^{\circ} 52-53$, octobre 2001

- HeinemanN A. W., «Finanzströme im Bundesstaat - Grundlagen für eine Föderalismusreform III in Deutschland ", ifo Dresden berichtet, $n^{\circ} 3 / 2013$

- HUBER B., «Föderaler Wettbewerb : Möglichkeiten und Grenzen », ZEW Wirtschaftsanalysen, vol. 49, 2000

- Institut deR DeUtSchen WiRTSchaft Köln (ed), Föderalismus in Deutschland. Ökonomische Analyse und Reformbedarf, Cologne, 2007

- KITTERER W., "Bundesstaatsreform und Zukunft der Finanzverfassung ", FiFo - CPE Discussion Papers, n07-3, 2007

- Länderfinanzen, Dossier in ZBW, Wirtschaftsdienst, $n^{\circ}$ 10/2014

- Peffekoven R., "Reform des Länderfinanzausgleichs und des Solidarpakts II. Statt grundlegender Reform fragwürdige Änderungen im Detail », Wirtschaftsdienst, 2001/VIII

- RAGNITZ J., «Altschuldenentlastung der Bundesländer : Verteilungswirkungen ausgewählter Vorschläge », ZBW, Wirtschaftsdienst, n 9/2014

- RENZSCH W., « Reform der Finanzverfassung zwischen ökonomischer Effizienz, bundesstaatlicher Funktionalität und politischer Legitimität », ZEW Wirtschaftsanalysen, vol. 49, 2000

- SACHVERSTÄNDIGENRAT ZUR BEgUTACHTUNG DER GESAMTWIRTSCHAFTLICHEN ENTWICKLUNG, Staatsverschuldung wirksam begrenzen. Expertise im Auftrag des Bundesministers für Wirtschaft und Technologie, mars 2007 ; et rapports annuels, principalement ceux de 2001, 2004, 2006, 2010 et 2014

- SteinPass P., "Sans le principe que le libre exercice de la responsabilité exige en contrepartie que celle-ci soit pleinement assumée, nulle communauté ne peut fonctionner durablement », 3101-2011 (www.cirac.u-cergy.fr/colloques_etudes_wp/interview4_fr.pdf)

-Scheller H., « Die Reform der Finanzverfassung », Aus Politik und Zeitgeschichte, 50/2006

- WISSENSCHAFTLICHER BEIRAT BEIM BUNDESMINISTERIUM Für WiRTSCHAFT, Zur Begrenzung der Staatsverschuldung nach Art. 115 GG und zur Aufgabe des Stabilitäts- und Wachstumsgesetzes, mars 2008 ; Zur finanziellen Stabilität des deutschen Föderalstaates, octobre 2005

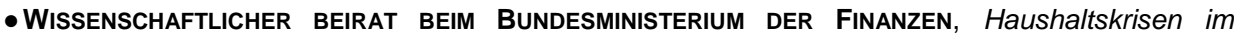
Bundesstaat, Gutachten, avril 2005, et Stellungnahme zum Finanzausgleichsurteil des Bundesverfassungsgerichts vom 11. November 1999

- www.bundesverfassungsgericht.de

-www.stabilitaetsrat.de. 